\title{
EFEITOS DE DOIS TIPOS DE ALONGAMENTO SOBRE A FLEXIBILIDADE DE MULHERES IDOSAS APÓS 12 SEMANAS DE TREINAMENTO FÍSICO GENERALIZADO
}

\author{
Nelson Hilario Carneiro ${ }^{1}$, Vinicius Flavio Milanez ${ }^{1}$, Eduardo Neris Carneiro ${ }^{1}$, Vanderlei Lopes da \\ Silva Junior ${ }^{1}$, André Castanho Freitas ${ }^{2}$, Lucas Rafael Santos Zangirolamo ${ }^{2}$, Luís Alberto Gobbo ${ }^{3}$ \\ Universidade do Oeste Paulista - UNOESTE, Curso de Educação Física, Presidente Prudente, SP. Instituto La Veritá, \\ Curso de Extensão e Pós-Graduação. Universidade Estadual Paulista - UNESP, Curso de Educação Física, Presidente \\ Prudente, SP. E-mail: nelson@unoeste.br
}

\section{RESUMO}

O objetivo deste estudo foi analisar os efeitos de dois métodos de alongamento sobre a flexibilidade em mulheres idosas após 12 semanas de treinamento físico generalizado (TFG). 41 mulheres com idade $\geq$ de 60 anos participaram do estudo, foram divididas em dois grupos, sendo grupo um (G1) que foi aplicado o alongamento de facilitação neuromuscular proprioceptivo (FNP) sendo cinco mulheres e o grupo dois (G2) foi aplicado o alongamento estático (AE) 36 mulheres. Todas as participantes fazem parte de um programa de treinamento físico generalizado. As avaliações foram realizadas antes da realização da intervenção (pré) e após a intervenção (pós). Foi realizada avaliação da massa corporal, da estatura, foi calculado o IMC, avaliação da flexibilidade com flexímetro e o teste de alcançar sentada. O programa de teve a duração de uma hora. O tratamento estatístico foi utilizado o Teste $\mathrm{t}$ de Student para amostras dependentes, considerando-se nível de significância $p<0,05$.

Palavras - chave: Alongamento, Facilitação neuromuscular proprioceptiva, Idosos, Flexibilidade.

\section{EFFECTS OF TWO TYPES OF STRETCHING ON OLDER WOMEN FLEXIBILITY AFTER 12 WEEKS OF PHYSICAL TRAINING WIDESPREAD}

\begin{abstract}
The objective of this study was to analyze the effects of two stretching methods on flexibility in older women after 12 weeks of general physical training (TFG). 41 women aged $\geq 60$ years participated in the study were divided into two groups, one group (G1) has been applied stretching proprioceptive neuromuscular facilitation (PNF) including five women and group two (G2) stretching was applied Static (AE) 36 women. All participants are part of a widespread program of physical training. The evaluations were conducted prior to the intervention (pre) and after the intervention (post). Was carried out assessment of body mass, height, BMI was calculated, evaluating the flexibility to fleximeter and to achieve sitting test. The program lasted for one hour. The statistical analysis we used the Student $t$ test for dependent samples, considering a significance level of $p<0.05$.
\end{abstract}

Keywords: stretching, proprioceptive neuromuscular facilitation, Seniors, Flexibility. 
INTRODUÇÃO

A força muscular, bem como a flexibilidade tem sido considerada as principais componentes da capacidade física, uma vez que auxiliar nas atividades de vida diária (AVD) para população idosa, postergando assim sua autonomia, logo, melhorando sua qualidade de vida. Muitos estudos tem sido realizado com o objetivo de amenizar ou até mesmo reverter a deterioração da estrutura neuromuscular, que esta diretamente associada com a perda da autonomia com avançar da idade. O envelhecimento está associado a modificações nos diferentes sistemas corporais, em especial, no sistema neuromuscular, provocando redução nos níveis de força, flexibilidade, resistência e potência muscular, componentes importantes da aptidão física, com impacto, sobretudo, para manutenção da autonomia e qualidade de vida em idosos ${ }^{1,2,3,4}$. Nesse sentido, para realização das atividades de vida diária (AVD) com mais vigor e menos fadiga, é importante a manutenção de níveis adequados de força e mobilidade articular é importante para não comprometer a eficácia na execução de diferentes movimentos envolvidos na ${ }^{1,5,6,7}$. Tendo em vista que exercícios com pesos tem sido recomendado para o desenvolvimento da força muscular, principalmente em idosos ${ }^{5}$, alguns estudos têm indicado que a participação regular em programas de treinamento com pesos (TP) pode contribuir também para o aumento da flexibilidade, em particular, nessa população ${ }^{1,8,9}$.

Entretanto, estudos anteriores analisaram apenas o impacto do TP sobre a flexibilidade, logo, programas de treinamento não consideraram diferentes tipos de alongamento. Desta maneira, não encontramos estudos analisando respostas em diferentes tipos de alongamento sobre a flexibilidade por meio de TP em idosos ${ }^{8,10,11}$.

Nesse sentido, o objetivo desse estudo foi analisar dois tipos de alongamento sobre a flexibilidade de mulheres idosas após 12 semanas de treinamento físico generalizado.

\section{MATERIAL E MÉTODOS}

\section{Sujeitos}

Participaram do estudo 41 mulheres idosas treinadas, com idade ( $\geq$ a 60 anos), que fizeram parte do projeto de extensão e pesquisa da Unoeste em parceria com o Grupo Athia, foram voluntárias para participarem desse estudo.

O programa de exercícios físicos generalizado foi desenvolvido em um salão próprio do Grupo Athia, As voluntarias deste estudo foram entrevistadas por meio de anamnese clínica, todas as participantes apresentaram um atestado médico, onde o médico atestou que as mesmas estavam consideradas aptas, sem restrição, para a prática de programas de exercícios físicos por 
médico cardiologista. Como critérios de exclusão foram adotados: a) não atingir $80 \%$ de presença ou, ainda, ter cinco faltas consecutivas as sessões de TP; b) contrair algum tipo de lesão ou doença que não permitam a execução plena do programa de treinamento ou participar dos períodos programados para a realização de medidas e avaliação do desempenho físico. A partir daí, as idosas foram aleatoriamente divididas em dois grupos, a saber: $G 1(n=05)$ o alongamento foi realizado por meio do método de facilitação neuromuscular proprioceptivo e para o $G 2(n=36)$ foi realizado o alongamento estático com duração de 20 segundos.

Todas as participantes após serem convenientemente informadas sobre a proposta do estudo (riscos, benefícios e procedimentos aos quais seriam submetidas) assinaram o Termo de Consentimento Livre e Esclarecido. Este estudo foi submetido pelo Comitê de Ética em Pesquisa envolvendo Seres Humanos da Universidade do Oeste Paulista, de acordo com as normas da Resolução 196/96 do Conselho Nacional de Saúde sobre pesquisa envolvendo seres humanos (Parecer 2284/2014).

\section{Antropometria e massa muscular}

A massa corporal foi mensurada em uma balança de plataforma digital, da marca Filizola, com escala de $0,1 \mathrm{~kg}$, e a estatura em um estadiômetro de madeira com escala de 0,1 cm, de acordo com os procedimentos descritos por ${ }^{12}$. A partir dessas medidas, calculamos o índice de massa corporal (IMC) por meio da razão entre a massa corporal e o quadrado da estatura, sendo a primeira expressa em quilogramas $(\mathrm{kg})$ e a segunda, em metros $(\mathrm{m})$.

\section{Avaliação da Flexibilidade}

Para avaliação da flexibilidade, foi adotado a flexão do tronco (FT). As medidas foram obtidas por meio de um flexímetro da marca Sanny ${ }^{\circledR}$ (American Medical do Brasil Ltda, São Bernardo do Campo, São Paulo, Brasil), com escala de um grau. Adicionalmente, o teste sentar-ealcançar sentada da bateria de testes da AAHPERD, foi aplicado como indicador da flexibilidade do quadril (FQ), de acordo com os procedimentos e as recomendações disponíveis na literatura ${ }^{13}$. O movimento de FT foi realizado na posição ortostática, as medidas foram obtidas de forma ativa em cada movimento, os movimentos foram realizados sem aquecimento prévio. Durante a realização das medidas as participantes foram orientadas a permanecerem na posição final até que a leitura ser efetuada. O maior escore obtido nas três medidas, em cada movimento articular, foi adotado como valor de referência. 
Durante as avaliações, o equipamento foi fixado no membro correspondente à articulação avaliada por meio de um velcro. Vale destacar que um único avaliador, com experiência, realizou tanto as medidas pré intervenção quanto após as 12 semanas de intervenção. As informações obtidas inicialmente não foram disponibilizadas para o avaliador no momento da reavaliação, na tentativa de evitar qualquer tipo de interferência que possa comprometer ou influenciar a qualidade dos dados.

\section{Programa de Exercício Físico Generalizado:}

Foi composto de 60 minutos, sendo 20 minutos de aquecimento e 40 minutos de treinamento com pesos. O aquecimento foi composto por exercícios aeróbios (dança) composto por coordenação motora, equilíbrio estático e dinâmico, propriocepção e flexibilidade, finalizando com alongamento. Além disso, foi dado ênfase no aquecimento anteriormente ao alongamento, desta maneira, foi realizado uma série de 15 chutes frontal, saindo da paralela, na sequencia foi realizado o alongamento, em seguida foi realizada uma segunda serie de 15 chutes frontal saindo da perna de trás, na posição anteroposterior, e novamente foi repetido o alongamento.

\section{Programa de Treinamento com Pesos}

O programa de TP foi realizado durante 12 semanas consecutivas, três vezes por semana. Todas as participantes foram supervisionadas por instrutores experientes na tentativa de uniformizar o padrão dos movimentos e fornecer segurança às voluntárias. O programa de treinamento foi dividido em $A$ e $B$, sendo que o Treinamento A (TA) consistiu da execução de três séries de nove a 12 RM em oito exercícios, na seguinte ordem: agachamento livre, Leg 45으, cadeira extensora, flexão de perna em pé apoiado em uma cadeira utilizando caneleiras, Stif, Panturrilha em pé, adução, abdução das pernas e abdominal, que foi realizado as segundas e sextas feiras. 0 Treinamento B (TB) consistiu na execução de três séries de nove a 12 RM em oito exercícios, na seguinte ordem: remada unilateral, tríceps testa, rosca simultânea, encolhimento, remada alta, elevação frontal, elevação lateral, rosca punho e crucifixo (peitoral). O intervalo de recuperação entre os exercícios foi de um a dois minutos. As cargas foram reajustadas periodicamente em cada exercício quando o número máximo de repetições programadas for atingido em duas sessões de treinamento consecutivas, que foi realizado nas quartas feiras. 
Alongamento Estático:

O AE foi aplicado de forma ativa e contínua, suave e gradativamente, aumentando a amplitude de movimento até alcançar o limite máximo, permanecendo nessa posição por cerca de 20 segundos $^{14}$.

\section{Facilitação Neuromuscular Proprioceptivo:}

A FNP foi realizado de forma passiva, o musculo alongado, primeiramente, o instrutor forçou a elevação da perna por cerca de 8 a 10 segundos, na sequência o aluno realizou uma contração máxima (forçando a perna a descer, por 8 segundos), na sequencia o aluno relaxou o músculo e o instrutor realizou uma força contraria, onde o instrutor segurou impedindo o movimento da mesma, após 6 a 8 segundos $^{14}$

\section{Análise estatística}

O tratamento estatístico foi utilizado o Teste t de Student para amostras dependentes, considerando-se nível de significância $p<0,05$.

\section{RESULTADOS}

A Tabela 1 apresenta os valores dos testes de flexibilidade por meio flexímetro Sanny e o teste de alcançar sentado da bateria da AAHPERD, pré e pós 12 semanas de Treinamento Generalizado ${ }^{15}$.

\begin{tabular}{|c|c|c|c|c|}
\hline Testes & Pré G1 & Pré G2 & Pós G1 & Pós G2 \\
\hline Flex (grau) & $89,4 \pm 17,2$ & $85,4 \pm 13,8$ & $108,2 \pm 10$ & $104,3 \pm 15,2$ \\
\hline $\begin{array}{c}\text { Alcançar } \\
\text { sentado } \\
\text { (centímetros) }\end{array}$ & $58,8 \pm 12,7$ & $56,3 \pm 9,3$ & $59,2 \pm 10,2$ & $58,1 \pm 9,5$ \\
\hline
\end{tabular}

\section{DISCUSSÃO}

Os achados do presente estudo foram que 12 semanas de treinamento generalizado, quando enfatizado o aquecimento localizado anteriormente as sessões de alongamento, independente do tipo de alongamento, foram suficiente para ganho da flexibilidade na população idosa. Entretanto, nenhuma diferença significativa foi encontrada quando comparamos os dois grupos. Sendo assim, os exercícios físicos generalizados realizados foram de encontro com processo de envelhecimento, mostrando efetividade no ganho da amplitude de movimentos, desta forma, postergando a capacidade funcional dessa população, uma vez que a flexibilidade é um importante componente da capacidade funcional. 
Nesse sentido, estudos realizados pela equipe do professor Gobbi corroboram com nossos estudos, encontraram interações Grupo x Tempo significativas para os movimentos de quadril direito e esquerdo (61 e 40\%, respectivamente) e; flexão do quadril esquerdo (9\%). O TP foi realizado durante oito semanas, com frequência de $3 \mathrm{X}$ semanais com 3 séries de 10 a $12 \mathrm{RM}$. Fizeram parte deste estudo 19 idosos, de ambos os sexos ${ }^{8}$.

CYRINO et al., 2004, estudaram o comportamento da flexibilidade de diferentes articulações após 10 semanas de treinamento com pesos. Os resultados das 10 primeiras semanas de prática de TP contribui efetivamente para a preservação ou melhoria dos níveis de flexibilidade observados no período pré-treinamento quando comparado com o momento pós, em diferentes articulações.

Outro estudo realizado pela equipe do prof. Cyrino, após 12 semana de prática de TP realizado duas e três vezes semanais em mulheres idosas destreinadas, encontraram melhoras em diferentes movimentos articulares e esses benefícios foram associados aos ganhos de força e massa muscular nessa população ${ }^{16}$.

Segundo Fatouros et al., ${ }^{10}$ outro fator que pode determinar os aumentos na flexibilidade para as diferentes articulações, em decorrência do TP, é a intensidade com a qual o exercício é realizado. 58 idosos participaram do estudo com duração de 12 semanas. Seus achados foram que os aumentos percentuais na flexibilidade das diferentes articulações apresentaram um comportamento intensidade dependente. De fato, o grupo treinado com baixa intensidade (40\% de 1-RM) obteve menor amplitude de aumento na flexibilidade (3-12\%), quando comparados aos grupos treinados com moderada intensidade (60\% de 1-RM) (6-22\%) e alta intensidade (80\% de 1RM) (8-28\%).

Desta maneira, a relevância do nosso estudo foi os diferentes métodos de alongamento em um programa de treinamento generalizado, uma vez que resultou em efeitos positivo sobre o processo de envelhecimento, onde além induzir a desaceleração da flexibilidade, ainda foi capaz de proporcionar ganho da mesma.

\section{CONCLUSÃO}

Concluímos que nossos achados sugerem que 12 semanas de treinamento generalizado em mulheres idosas já treinadas, pode contribuir na manutenção ou até mesmo no ganho da flexibilidade, independente o tipo de alongamento. 


\section{REFERÊNCIAS}

1. Carneiro NH, Ribeiro AS, Nascimento MA, Gobbo LA, Schoenfeld BJ, Achour Junior AA, Gobbi S, Oliveira AR, Cyrino ES. et al. Effects of diferente resistance training frequencies on flexibility in older women. Clinical Interventions in Aging. 2015:10 531-538. DOI

2. Carneiro NH, Silva COV. Comparação das capacidades funcionais de mulheres idosas praticantes de exercícios físicos durante oito semanas. Colloquium Vitae. Vol. 6. N. Especial, juldez, 2014, p. 29-35. ISSN: 1984-6436. DOI 10.5747/cv.2014.v06.nesp.000231

3. Carneiro NH, Garcia Junior JR. Efeitos da prática da capoeira adaptada para terceira idade. Colloquium Vitae. 2009;1:17-24. http://dx.doi.org/10.5747/cv.2009.v01.n1.v003

4. Levinger I, Goodman C, Hare DL, Jerums G, Selig S. The effect of resistance training on functional capacity and quality of life in individuals with high and low numbers of metabolic risk factors. Diabetes Care. 2007;30(9):2205-10. http://dx.doi.org/10.2337/dc07-0841

5. Vale RGS, Barreto ACG, Novaes JS, Dantas EHM. Efeitos do treinamento resistido na força máxima, na flexibilidade e na autonomia funcional de mulheres idosas. Rev Bras Cineantropom Desempenho Hum. 2006;8(4):52-8.

6. American College of Sports Medicine. Position stand: progression models in resistance training for healthy adults. Med Sci Sports Exerc. 2009;41(3):687-708. http://dx.doi.org/10.1249/MSS.0b013e3181915670

7. American College of Sports Medicine. Position stand: quantity and quality of exercise for developing and maintaining cardiorespiratory, musculoskeletal, and neuromotor fitness in apparently healthy adults: guidance for prescribing exercise. Med Sci Sports Exerc. 2011;43(7):1334-59. http://dx.doi.org/10.1249/MSS.0b013e318213fefb

8. Gonçalves R, Gurjão ALD, Gobbi S. Efeitos de oito semanas de treinamento de força na flexibilidade de idosos. Rev Bras Cineantropom Desempenho Hum. 2007;9(2):145-53.

9. Seco J, Abecia LC, Echevarria E, Barbero I, Torres-Unda J, Rodriguez V, et al. A long-term physical activity training program increases strength and flexibility, and improves balance in older adults. Rehabil Nurs. 2012;38(1):37-47. http://dx.doi.org/10.1002/rnj.64

10. Fatouros IG, Kambas A, Katrabasas I, Leontsini D, Chatzinikolaou A, Jamurtas AZ, et al. Resistance training and detraining effects on flexibility performance in the elderly are intensity dependent. J Strength Cond Res. 2006;20(3):634-42. http://dx.doi.org/10.1519/00124278$\underline{200608000-00027}$

11. Gobbi S, Villar R, Zago AS: Bases Teóricas - Práticas do condicionamento físico, Guanabara Koogan S.A., 2005.

12. Gordon CC, Chumlea WC, Roche AF. Stature, recumbent length, and weight. In: Lohman TG, Roche $A F$, Martorell $R$, editors. Anthropometric standardizing reference manual. Champaign: Human Kinetics Books, 1988:3-8.

13. Achour JA. Manual de instruções: avaliando a flexibilidade. Londrina: Midiograf; 1997. 
14. Carneiro NH. Capoeira \& Alongamento. Gráfica Cipola. 2013.

15. Zago AS, Gobbi S. Valores normativos da aptidão funcional de mulheres de 60 a 70 anos. Revista Brasileira de Ciência e Movimento, Brasília, v.11, n.2, p.77-86, 2003.

16. Cyrino ES, Oliveira AR, Leite JC, Porto DB, Dias RMR, Segantin AQ, et al. Comportamento da flexibilidade após 10 semanas de treinamentos com pesos. Rev Bras Med Esporte. 10(4):233-7. 2004. http://dx.doi.org/10.1590/S1517-86922004000400001 